\title{
Politique
}

\section{Les traditions de recherche en science politique}

\section{Réjean Landry}

Numéro 23, hiver 1993

Tendances de la science politique au Québec

URI : https://id.erudit.org/iderudit/040745ar

DOI : https://doi.org/10.7202/040745ar

Aller au sommaire du numéro

Éditeur(s)

Société québécoise de science politique

ISSN

0711-608X (imprimé)

1918-6584 (numérique)

Découvrir la revue

Citer cet article

Landry, R. (1993). Les traditions de recherche en science politique. Politique, (23), 7-19. https://doi.org/10.7202/040745ar d'utilisation que vous pouvez consulter en ligne.

https://apropos.erudit.org/fr/usagers/politique-dutilisation/ 


\title{
LES TRADITIONS DE RECHERCHE EN SCIENCE POLITIQUE
}

\author{
Réjean Landry
}

Université Laval

Tout le monde discute de politique. Ces discussions présentent trois traits communs. II s'agit souvent de discussions non rigoureuses qui ne reposent pas sur l'utilisation systématique de théories, de méthodes et de techniques d'analyse politique. II s'agit aussi de discussions basées habituellement sur des exemples factuels plutôt que sur des données ayant été soumises à des tests de validité et de fiabilité. II s'agit enfin de discussions généralement dépourvues de perspective historique sur les comportements, les institutions et les services publics.

Les travaux de recherche en science politique visent à combler une partie de ces lacunes par l'utilisation de la méthode scientifique. Celle-ci repose essentiellement sur trois catégories d'outils : des théories, des techniques de collecte de données et des techniques d'analyse et d'interprétation de données. Les théories constituent des ensembles de propositions s'enchaînant de façon déductive et desquelles dérivent les hypothèses de recherche. Quant aux techniques de collecte des données, elles concernent les procédures utilisées pour observer des faits. Ces procédures doivent être suffisamment rigoureuses et systématiques pour permettre à d'autres chercheurs qui le désireraient de reproduire les mêmes observations. Finalement, les techniques $d$ 'analyse et d'interprétation des données concernent la confrontation des faits prédits par une théorie avec les faits observés à l'étape de la collecte de données. La confrontation requiert le recours à des techniques de contrôle de variables dont les procédures appartiennent aux statistiques et à la méthode expérimentale. Toutes les sciences, qu'il s'agisse des sciences de la nature, des sciences de la vie ou des sciences sociales procèdent de 
la même manière. II n'existe qu'une seule façon de faire de la science et elle repose sur l'utilisation de la méthode scientifique.

La politique concerne les décisions dont les effets s'imposent à l'ensemble de la collectivité alors que la science politique étudie les règles qui déterminent ces décisions, de même que les conséquences qui en résultent tant pour l'individu que pour la collectivité.

De façon plus spécifique, on peut dire que la science politique étudie les arrangements institutionnels - structures institutionnelles et règles de décision - utilisés pour la prise de décisions imposées aux individus composant des collectivités. C'est là l'objet d'étude le plus ancien de la science politique, traditionnellement assimilé à l'étude des institutions. Cette conception de la discipline remonte à Aristote qui s'intéressait à l'analyse comparative des constitutions des cités grecques. Elle a perdu de son importance avec l'apparition des techniques statistiques et de I'informatique qui, à partir de la seconde guerre mondiale, a incité les politologues à étudier les autres facteurs qui influencent les décisions politiques des citoyens et des politiciens. À leur tour, ces travaux d'inspiration behavioriste ont fait percevoir la science politique comme étant l'étude des phénomènes de pouvoir politique. Cette seconde façon de voir la discipline a orienté les méthodes de la science du côté des techniques de collecte de données et des techniques d'analyse et d'interprétation des données. À partir des années 1960, on a souligné de plus en plus les déficiences théoriques de cette tradition de recherche. La volonté d'utiliser à tout prix les méthodes de la science, notamment les statistiques, avait en effet conduit beaucoup de chercheurs à ne retenir pour objet d'étude que des phénomènes quantifiables, ou encore, des phénomènes pour lesquels on disposait déjà de données quantitatives. II en est résulté des théories réductionnistes qui ne rendaient plus compte de l'impact des arrangements institutionnels sur les décisions des individus.

Les années 1980 et 1990 constituent une période de transition où l'on assiste à une résurgence de l'étude des institutions tant au niveau des structures que des règles de 
décision. Les études à caractère holistique, qui s'inscrivent dans l'école du nouvel institutionnalisme (March et Olsen, 1989), et celles de l'école du public choice sur les règles de décision (Ostrom, 1990; 1993) contribuent à redonner de l'importance et de la vigueur à l'étude des institutions.

Au même moment, les travaux d'économistes comme Samuelson, Downs et Olson ont sensibilisé les politologues à l'impact que les attributs des biens, publics ou privés, exercent sur les choix des individus. Le magistral ouvrage d'Olson (1965) sur la logique de l'action collective, qui démontre que des individus rationnels ne sont pas incités à contribuer à des groupes qui produisent des biens publics, a eu une très grande influence.

La science politique actuelle a donc pour objet d'étude trois catégories de variables en forte interaction :

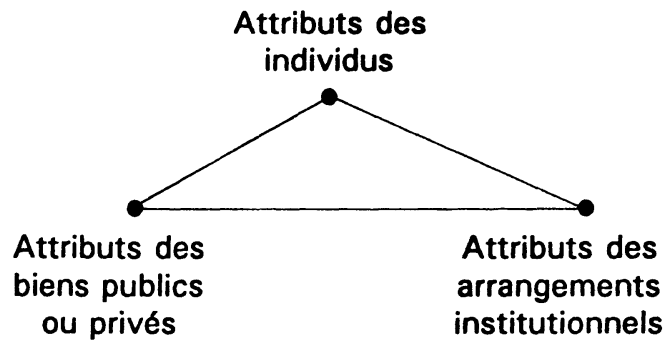

Des trois catégories de variables et de l'interaction qui les soude découlent l'analyse normative et l'analyse positive. $L$ 'analyse normative s'intéresse aux valeurs et aux normes qui devraient prévaloir lors de la préparation des politiques publiques et fait l'examen des conséquences éthiques des choix dans le domaine des politiques publiques. Elle relève donc de l'éthique et de la philosophie politique. Elle se penche, par exemple, sur ce type de questions : les interventions de l'État devraient-elles promouvoir une extension de la liberté individuelle au détriment de la protection de l'égalité de résultats entre les individus ?

$L$ 'analyse positive concerne des énoncés du genre "si $X$, 


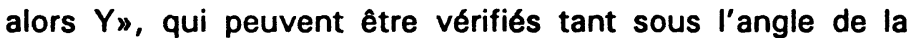
logique que celui de l'observation empirique; par exemple, elle s'intéresse à la logique de l'offre et de la demande de politiques ou à la logique de la compétition entre les partis.

Ces deux types d'analyses se conjuguent souvent pour faire émerger un troisième type, soit /'analyse de résolution de problèmes, qui étudie et identifie les solutions possibles des problèmes politiques : la façon, par exemple, dont le gouvernement peut améliorer les prestations accordées aux assistés sociaux sans inciter les assistés à ne pas réintégrer le marché du travail.

Tableau I : Les trois types d'analyses de la science politique

\begin{tabular}{|c|c|c|}
\hline Normative & Positive & $\begin{array}{c}\text { Résolution } \\
\text { de } \\
\text { problèmes }\end{array}$ \\
\hline $\begin{array}{c}\text { Actions et valeurs } \\
\text { qui devraient } \\
\text { prévaloir }\end{array}$ & $\begin{array}{c}\text { Consistance logique } \\
+ \\
\text { observation empirique }\end{array}$ & $\begin{array}{c}\text { Solutions } \\
\text { possibles : } \\
\text { normatif } \\
+ \\
\text { positif }\end{array}$ \\
\hline $\begin{array}{l}\text { Philosophie } \\
\text { politique }\end{array}$ & Sociologie politique & $\begin{array}{l}\text { Politiques } \\
\text { publiques }\end{array}$ \\
\hline
\end{tabular}

Bien que les spécialistes de la science politique tendent à adopter l'une ou l'autre de ces trois perspectives, il importe de noter que les interrelations harmonieuses qui existent entre ces trois éléments font de la science politique une discipline unifiée et cohérente. 
Tableau II : Les trois types d'analyses de la science politique et leurs interrelations.

\begin{tabular}{|c|c|c|c|}
\hline Analyse & $\begin{array}{l}\text { Valeurs } \\
\text { politiques }\end{array}$ & $\begin{array}{c}\text { Phénomènes } \\
\text { politiques }\end{array}$ & $\begin{array}{c}\text { Solutions } \\
\text { possibles } \\
\text { des } \\
\text { problemes }\end{array}$ \\
\hline Normative & $\begin{array}{l}\text { Quelles } \\
\text { valeurs } \\
\text { politiques } \\
\text { devraient } \\
\text { prévaloir } \\
\text { dans les } \\
\text { choix } \\
\text { collectifs? }\end{array}$ & $\begin{array}{l}\text { Comment les ac- } \\
\text { teurs politiques } \\
\text { devraient-ils se } \\
\text { comporter? } \\
\text { (choix, décisions) }\end{array}$ & $\begin{array}{l}\text { Quelles } \\
\text { caractéristi- } \\
\text { ques les } \\
\text { politiques } \\
\text { publiques } \\
\text { devraient- } \\
\text { elles avoir? }\end{array}$ \\
\hline Positive & $\begin{array}{l}\text { Quelles } \\
\text { valeurs pré- } \\
\text { valent dans } \\
\text { les choix } \\
\text { collectifs? }\end{array}$ & $\begin{array}{l}\text { Comment les ac- } \\
\text { teurs politiques se } \\
\text { comportent-ils } \\
\text { (choix) dans la } \\
\text { réalité? }\end{array}$ & $\begin{array}{l}\text { Quelles sont } \\
\text { les caracté- } \\
\text { ristiques des } \\
\text { politiques } \\
\text { publiques en } \\
\text { vigueur? }\end{array}$ \\
\hline $\begin{array}{l}\text { Résolu- } \\
\text { tion } \\
\text { de } \\
\text { problèmes }\end{array}$ & $\begin{array}{l}\text { Quelles } \\
\text { valeurs } \\
\text { pourrait-on } \\
\text { faire } \\
\text { prévaloir } \\
\text { dans les } \\
\text { choix collec- } \\
\text { tifs? }\end{array}$ & $\begin{array}{l}\text { Comment les ac- } \\
\text { teurs politiques } \\
\text { pourraient-ils se } \\
\text { comporter? } \\
\text { (choix, décisions) }\end{array}$ & $\begin{array}{l}\text { Quelles sont } \\
\text { les caracté- } \\
\text { ristiques des } \\
\text { politiques } \\
\text { publiques qui } \\
\text { peuvent être } \\
\text { formulées et } \\
\text { appliquées } \\
\text { de façon } \\
\text { réaliste? }\end{array}$ \\
\hline
\end{tabular}

L'émergence de la science politique comme discipline scientifique s'est effectuée progressivement, au fil des siècles, en même temps qu'une séparation de plus en plus étanche s'établissait entre l'analyse normative et l'analyse positive. Cette séparation s'est confirmée de façon plus nette 
au XIX' siècle avec l'utilisation des outils de la méthode scientifique dans les sciences sociales.

\section{Deux unités d'analyse}

La description, l'explication et la prédiction des décisions (interventions de l'État) qui s'imposent à tous les membres d'une collectivité peuvent être envisagées suivant qu'elles adoptent la structure ou l'individu comme unité d'analyse. Du point de vue de la construction d'une théorie, l'unité d'analyse est l'élément à partir duquel un chercheur formule des propositions qui décrivent, expliquent ou prédisent les décisions des individus ou des collectivités.

Certains politologues soutiennent que la structure globale de la société est l'élément de base à partir duquel doivent s'élaborer les théories. Ils considèrent que les structures institutionnelles globales (par exemple : monarchie, aristocratie, etc.) et les structures sociales globales (par exemple : les types de nations, les types de classes sociales, les femmes, les jeunes, les handicapés, etc.) constituent les unités d'analyse à la base de la science politique. Suivant cette perspective, il faut partir des structures institutionnelles globales et des structures sociales globales pour expliquer les décisions et les actions des individus. Cette conception implique que les structures globales ont une existence en soi, indépendante des individus, et qu'elles composent des totalités réelles observables empiriquement. II faut donc expliquer les parties par le tout. Les structures globales ne peuvent être appréhendées à partir d'éléments isolés. II faut commencer par examiner l'ensemble des structures globales pour être en mesure d'en comprendre les éléments. C'est la logique du holisme méthodologique.

Les théories qui utilisent les classes sociales ou la nation comme unité d'analyse sont de bonnes illustrations de cette façon de voir. La structure globale a constitué l'unité d'analyse dominante de la science politique ces dernières années. Les théories structuro-fonctionnalistes et marxistes qui se sont partagé, jusqu'à tout récemment, une grande part de l'espace théorique en science politique représentent les principales traditions de recherche des dernières décennies, 
lesquelles s'appuyaient sur la structure globale comme unité d'analyse, l'individu n'étant alors rien d'autre qu'un élément non autonome remplissant une fonction précise dans la collectivité.

L'erreur du holisme méthodologique, illusion méthodologique, consiste à attribuer les caractéristiques des individus à des structures et à des collectivités. Or, les structures et les collectivités ne constituent pas des êtres vivants. Elles ne font strictement rien. Ainsi, la nation québécoise est incapable de téléphoner chez Pizza Hut pour commander une pizza géante. Les structures et les collectivités sont incapables de choisir et d'agir indépendamment des actions et des décisions des individus qui les composent.

\section{L'individualisme méthodologique}

Dans les sociétés occidentales, c'est à partir du XIX siècle que l'individu a acquis le statut d'entité autonome possédant des droits. L'extension du droit de vote à toutes les catégories de citoyens et, au cours du XX' siècle, l'extension des droits sociaux ont donné naissance à des philosophies et à des théories qui accordent une place centrale à l'individu. Les théories qui prennent l'individu comme unité $d^{\prime}$ analyse $s$ 'inscrivent dans la logique de I'individualisme méthodologique. Les tenants de cette logique postulent qu'il est impossible d'observer directement les décisions d'une collectivité et que les décisions collectives résultent de l'agrégation des décisions prises par les individus. IIs en déduisent donc que l'individu doit constituer l'unité $d$ 'analyse de la science politique, que l'individu est le décideur ultime dans une collectivité. La logique de l'individualisme méthodologique consiste donc à partir des décisions et actions des individus pour décrire, expliquer et prédire les décisions et les actions de la collectivité. L'individualisme méthodologique fait de la société le résultat de l'addition des initiatives individuelles et de leurs interactions, et non une entité englobante conditionnant la moindre action humaine. II n'y a pas de société en soi et tout ce qui se fait dans une société est accompli par les individus. 
II est important de ne pas confondre l'individualisme méthodologique avec l'individualisme philosophique qui renvoie à un ensemble de valeurs en vertu desquelles toute intervention de l'État est indésirable. Les individus devraient être laissés libres de poursuivre leurs objectifs avec le minimum d'interférence de l'État.

\section{Approches analytiques}

Une approche analytique renvoie au point de vue adopté et à la méthode utilisée pour étudier des phénomènes. À différentes périodes, les tenants de l'analyse positive et normative ont développé de nouvelles approches qui ont contribué au développement de la science politique. Toutes les approches analytiques possèdent certaines caractéristiques communes : elles s'appuient toutes sur des postulats qui établissent les fondations d'un cadre d'analyse et inspirent des théories et des hypothèses qui contribuent àl'avancement des connaissances dans le champ propre à l'analyse positive et normative. À l'inverse, les approches analytiques se démarquent les unes des autres par des différences fondamentales qui déterminent leur validité et leur utilité au niveau analytique. Certaines sont plus développées et plus fines; d'autres sont plus simples et plus rudimentaires. Certaines comportent plusieurs éléments conceptuels et méthodologiques; d'autres mettent l'accent sur quelques concepts seulement.

La science politique contemporaine se caractérise par la présence parallèle de quatre approches dominantes. Celles-ci résultent de l'interaction de la perspective d'analyse (positive, normative ou de résolution de problèmes) avec l'unité d'analyse, l'individu ou les structures. Aucune ne bénéficie d'une situation de monopole. En fonction de sa formation, mais surtout de ses préférences (valeurs) personnelles, chaque politologue tend à opter pour l'une ou l'autre de ces quatre approches : libérale-rationnelle, behavioriste, structuro-fonctionnaliste et politico-économique. 
Tableau III : Types d'approches analytiques

\begin{tabular}{|l|l|l|}
\hline \multicolumn{1}{|c|}{ Analyse } & \multicolumn{2}{|c|}{ Unités d'analyse } \\
\hline & \multicolumn{1}{|c|}{ Individus } & \multicolumn{1}{c|}{ Structures } \\
\hline Normative & $\begin{array}{l}\text { Libérale- } \\
\text { rationnelle }\end{array}$ & Politico-économique \\
\hline Positive & Behavioriste & $\begin{array}{l}\text { Structuro- } \\
\text { fonctionnaliste }\end{array}$ \\
\hline $\begin{array}{l}\text { Résolution de } \\
\text { problèmes }\end{array}$ & $\begin{array}{l}\text { Libérale- } \\
\text { rationnelle } \\
\text { et } \\
\text { behavioriste }\end{array}$ & $\begin{array}{l}\text { Politico-économique } \\
\text { et } \\
\text { structuro- } \\
\text { fonctionnaliste }\end{array}$ \\
\hline
\end{tabular}

Chacune des sous-sections de ce tableau pourrait contenir d'autres approches. Toutefois, celles qui sont mentionnées renvoient probablement aux perspectives qui exercent l'influence la plus déterminante sur les travaux actuels en science politique. En outre, bien que chaque politologue ait tendance à opter pour l'une de ces approches, il existe probablement peu de politologues dont la perspective analytique corresponde totalement à une seule approche.

Les articles de ce numéro de la Revue québécoise de science politique démontrent que l'analyse positive tend à dominer la science politique pratiquée au Québec, bien que l'analyse normative et l'analyse de résolution de problèmes occupent une place de plus en plus importante dans la recherche.

Le bilan de l'étude des institutions électorales et parlementaires, dressé par Louis Massicotte, montre que les politologues du Québec ont tendance à analyser les institutions électorales sous l'angle des règles microinstitutionnelles alors qu'ils cherchent plutôt à appréhender les institutions parlementaires sous l'angle des structures institutionnelles. L'auteur souligne avec une grande justesse que les politologues québécois ont produit très peu de travaux concernant les institutions électorales et parlementaires 
d'autres provinces et d'autres pays. Louis Massicotte invite les analystes à enrichir leur programme de recherche par l'ajout $d$ 'institutions d'autres provinces et $d$ 'autres pays.

Le bilan tracé par Vincent Lemieux montre que l'étude des partis politiques renvoie principalement à des perspectives d'analyse positive et que les chercheurs se sont surtout attachés à l'étude du comportement des partis et à l'examen des structures organisationnelles des partis. Vincent Lemieux note que les politologues québécois ont accordé quasiment toute leur attention à l'étude des partis politiques provinciaux du Québec, au point où même les partis politiques fédéraux du Québec n'ont à peu près pas été étudiés. II invite les chercheurs à accorder désormais plus d'attention aux structures des partis, à la contribution des partis aux politiques publiques, de même qu'aux rôles des partis en tant qu'instruments de médiation entre les publics et le gouvernement.

James lain Gow passe en revue les travaux de recherche québécois qui concernent l'administration publique. II y distingue quatre grandes traditions de recherche : le paradigme classique pluraliste qui s'intéresse au rôle de l'État, le paradigme juridique qui met l'accent sur l'analyse des normes juridiques, le paradigme du management préoccupé par l'utilisation optimale des ressources, et finalement, le paradigme sociologique qui s'intéresse aux macrophénomènes. L'article de James lain Gow indique chez les spécialistes de l'administration publique, plus que chez les autres experts de la science politique, une alternance entre I'analyse positive et I'analyse normative.

L'article de Louis M. Imbeau et Guy Lachapelle sur les études comparatives des politiques publiques des provinces canadiennes aborde deux questions cruciales : les politiques publiques tendent-elles à converger ou non ? Quels facteurs politiques déterminent les politiques provinciales ? Dans cette littérature, la tendance à la convergence est généralement attribuée à la similarité des défis sociaux et économiques auxquels les gouvernements font face. L'école des déterminants, pour sa part, postule que les politiques publiques ne dépendent pas seulement de facteurs sociaux et économiques mais également de facteurs politiques associés 
à diverses caractéristiques des partis, notamment leur orientation idéologique. Louis M. Imbeau et Guy Lachapelle notent, comme le soulignent plusieurs autres articles de ce numéro, que les chercheurs québécois ont jusqu'à maintenant consacré l'essentiel de leurs efforts à l'étude de la spécificité québécoise plutôt qu'à la comparaison du Québec avec d'autres provinces et d'autres pays. Les auteurs notent que, dans le domaine des études comparatives, il n'existe pas de véritable école au Québec.

L'article de Louis Bélanger, Guy Gosselin et Gérard Hervouet, consacré aux travaux de recherche sur les relations internationales du Québec, distingue les travaux recensés suivant deux approches analytiques propres à ce secteur : I'approche géopolitique et l'approche de politique étrangère. Les études sur les relations internationales du Québec ont mis l'accent sur trois catégories de déterminants : le contexte interne, le contexte canadien et le contexte international. Contrairement aux études sur les politiques publiques, pour lesquelles les chercheurs disposent d'indicateurs, de statistiques officielles et de sondages sur les déterminants des politiques, les travaux sur les relations internationales ont nécessité le développement d'indicateurs spécifiques, cela dans un monde où les statistiques officielles et les données de sondages semblent assez rares.

L'article qui clôt le présent numéro de la revue concerne les études réalisées au Canada anglais sur la question du Québec. L'auteur, Serge Denis, y fait une méta-analyse des études normatives réalisées par des politologues et des économistes du Canada anglais. Cette méta-analyse fait ressortir avec éloquence la grande diversité des critères utilisés pour évaluer et juger différentes dimensions de la politique québécoise. Ce faisant, Serge Denis a ouvert un champ d'analyse qui devrait être développé.

\section{Conclusion}

Le bilan des traditions de recherche, que dresse la Revue québécoise de science politique, ne rend évidemment pas compte de toute la diversité des traditions qui se sont développées au Québec. Les travaux mentionnés ici 
s'inscrivent principalement dans les approches analytiques positives relevant du behaviorisme et du structurofonctionnalisme. Ainsi, ce bilan ne rend pas compte des approches analytiques normatives se situant dans la tradition libérale-rationnelle ou dans celle de l'économie politique. L'approche libérale-rationnelle n'a jusqu'à maintenant pas inspiré beaucoup de travaux de science politique au Québec alors que l'approche de l'économie politique, à l'image du déclin du socialisme et de la gauche, est probablement en train de réviser les éléments explicatifs et prédictifs de son paradigme.

En guise de note finale, il convient de souligner à nouveau, à la suite des auteurs de ce numéro de la Revue québécoise de science politique, que les politologues francophones du Québec ont jusqu'à maintenant consacré l'essentiel de leurs efforts à l'analyse des spécificités québécoises. Un nombre grandissant de politologues québécois s'intéressent maintenant à la politique comparée. On peut voir là un créneau susceptible de permettre aux politologues québécois de produire des travaux exportables sur le marché international des publications en science politique. La mondialisation et la globalisation des marchés ne concernent pas seulement les entreprises québécoises, elles concernent également les spécialistes de la science politique dont les productions seront de plus en plus appelées à rivaliser avec les meilleures productions scientifiques d'autres pays. 
Bibliographie

March, J.G. et J.P. Olsen, Rediscovering Institutions, New York, The Free Press, 1989.

Olson, M., The Logic of Collective Action, Cambridge, Harvard University Press, 1965.

Ostrom, E., Governing the Commons, Cambridge, Cambridge University Press, 1990.

Ostrom, E., L. Schroder et S. Wynne, Institutional Incentives and Sustainable Development, Boulder, The Westview Press, 1993. 